\section{A mortalidade materna nas \\ capitais brasileiras: algumas \\ características e estimativa de um \\ fator de ajuste*}

\section{Maternal mortality in Brazilian State Capitals: some characteristics and estimates for an adjustment factor}

\author{
Ruy Laurenti \\ Centro Brasileiro de Classificação de Doenças \\ Departamento de Epidemiologia \\ Faculdade de Saúde Pública \\ Universidade de São Paulo \\ Av. Dr. Arnaldo, 715 \\ 01246-904 - São Paulo, SP \\ laurenti@usp.br
}

\section{Maria Helena Prado de Mello Jorge \\ Departamento de Epidemiologia \\ Faculdade de Saúde Pública \\ Universidade de São Paulo}

\section{Sabina Léa Davidson Gotlieb}

Departamento de Epidemiologia

Faculdade de Saúde Pública

Universidade de São Paulo

\section{Resumo}

A mortalidade materna pode ser considerada um excelente indicador de saúde, não só da mulher, mas da população geral; mostra, também, iniqüidades. A redução da mortalidade materna é uma das principais metas, estando também incluída nas Metas do Desenvolvimento do Milênio da ONU. Objetivo: Conhecer a qualidade da informação da mortalidade de mulheres de 10 a 49 anos, e estimar a razão de mortalidade materna (RMM) e os fatores de ajuste para os dados oficiais, no conjunto das capitais de estados brasileiros e Distrito Federal. Metodologia: Adotou-se a metodologia RAMOS (a partir da declaração de óbito, entrevista no domicílio da mulher falecida, com preenchimento de questionário, sobre variáveis demográficas, epidemiológicas, clínicas e de acesso a serviços; seguiam-se consultas a prontuários médicos hospitalares e a laudos de autópsia). Após o resgate da informação, pôde ser feita análise das reais causas básicas, terminais e associadas. A população de estudo foi estimada em 7.332 mortes de mulheres de 10 a 49 anos, ocorridas no primeiro semestre de 2002, sendo 239 óbitos por causas maternas. A RMM foi de 54,3 por cem mil nascidos vivos (n.v.), no conjunto de capitais, variando entre 42 por cem mil n.v. no Sul, e 73,2 por cem mil n.v. no Nordeste. O fator de ajuste para o conjunto das capitais brasileiras foi igual a 1,4; para as regiões (considerando apenas as capitais), variaram entre 1,08 na Região Norte e 1,83 na Região Sul. As mortes obstétricas diretas corresponderam a $67,1 \%$, mostrando que assistência ao pré-natal, ao parto e ao puerpério deve ser aprimorada.

Palavras-chave: Mortalidade. Saúde da mulher. Epidemiologia. Metodologia RAMOS. Fator de ajuste.

* Parte do "Estudo de mortalidade de mulheres de 10 a 49 anos, com ênfase na mortalidade materna". Financiamento do Ministério da Saúde, OPS/OMS, USP e CNPq. 
Abstract

Introduction: There is criticism as to the heterogeneity and reliability of mortality data in Brazilian Regions. However, official mortality statistics of State Capitals are more accurate and have adequate coverage. Reduction of maternal mortality is one of the world's major goals and it is measured by the Maternal Mortality Ratio. International agencies have been estimating that the Brazilian ratio is very high. An investigation was carried out to know the actual value. Objective: The aim was to calculate the maternal mortality ratio for Brazilian Capitals and the Federal District and estimate an adjustment factor for official data. Methodology: A total of 7,332 deaths that occurred in the first semester of 2002 in women from 10 to 49 years of age living in these areas were investigated, using the RAMOS methodology (household interviews and investigation of medical and hospital records and autopsies.). Results: The maternal mortality ratio calculated was 54.3 per 100,000 live born, ranging between 73.2 in the North State Capitals and 42 per 100,000 live born in the South. The lowest adjustment factors were 1.08 in the North Region, and 1.10 in the Middle West State Capitals. The highest values were 1.76 and 1.83 in the Northeast and South Regions. Considering the set of Brazilian State Capitals, the adjustment factor estimated was equal to 1.4. The maternal mortality ratios observed were lower than WHO, UNICEF and UNFPA values. Direct obstetric maternal deaths accounted for $67.1 \%$ of the total, thus indicating that pre-natal and childbirth care must be improved.

Key Words: Maternal mortality. Adjustment factor. RAMOS methodology.

\section{Introdução}

A mortalidade materna pode ser considerada um excelente indicador de saúde, não apenas da mulher, mas da população como um todo. Por outro lado, é também um indicador de iniqüidades, pois não somente é elevada em áreas subdesenvolvidas ou em desenvolvimento, quando comparada aos valores de áreas desenvolvidas, quanto, mesmo nestas, existem diferenças entre os diferentes estratos socioeconômicos.

A Organização Mundial de Saúde-OMS define morte materna, segundo expresso na Classificação Internacional de Doenças- 10a Revisão (CID-10) ${ }^{1}$, como a morte de uma mulher durante a gestação ou dentro de um período de 42 dias após o término da gestação, independente da duração ou da localização da gravidez, devido a qualquer causa relacionada com ou agravada pela gravidez ou por medidas em relação a ela, porém não devida a causas acidentais ou incidentais.

Em publicação da WHO, UNICEF e UNFPA $^{2}$, é comentado que a redução da mortalidade materna é uma das principais metas discutidas em conferências internacionais atuais, estando, também, incluída nos Objetivos de Desenvolvimento do Milênio das Nações Unidas ${ }^{3}$. A avaliação desta redução não é fácil, dado que a mensuração da mortalidade materna é relativamente complexa. Isto ocorre tanto pela inexistência de dados para algumas regiões e países, como pela parcial fidedignidade da informação, mesmo naquelas áreas onde há declaração médica da causa da morte e bom sistema de registros vitais.

No Brasil, a mortalidade materna tem sido motivo de preocupação das autoridades de saúde, em nível federal, estadual e municipal. Em grande número de municípios, há "Comissões de Estudo e Prevenção de Mortes Maternas"4, que investigam os casos de morte materna declarada ou suspeita, sendo que, em muitos deles, são pesquisadas todas as mortes de mulheres em idade fértil. No Estado do Paraná, por exemplo, as investigações das mortes femininas de 10 a 49 anos são realizadas em todos os seus municípios e os 
dados são publicados individualizados e para o Estado como um todo. Assim, é possível calcular a razão de mortalidade materna corrigida, bem como um fator de ajuste ${ }^{5}$.

No Brasil, há um bom Sistema de Informações sobre Mortalidade, gerido pelo Ministério da Saúde (SIM/MS), implantado desde meados da década de 70 , e cujo número total de eventos captados está próximo a um milhão de óbitos/ano. Por meio de métodos demográficos, estima-se que a cobertura do SIM/MS seja de cerca de $85 \%$. Essa sub-informação concentra-se, quase que exclusivamente, nas Regiões Norte e Nordeste do país. Nos Estados do Sul e Sudeste, onde vive a maior parte da população brasileira $(57,4 \%)$, a abrangência é muito boa, aproximando-se de $100 \%$, particularmente nas suas capitais ${ }^{6}$.

Entretanto, mesmo nas regiões com boa ou excelente cobertura do registro, sabe-se que a declaração de uma causa materna como causa de morte não é totalmente exata ${ }^{7}$.

Para conhecer a verdadeira magnitude da taxa ou razão de mortalidade materna (RMM) e calcular um fator de ajuste para os dados oficiais, a melhor forma é obtê-los pelo método de pesquisa chamado "Reproductive Age Mortality Study” (RAMOS) ${ }^{8}$. Aplicar esta metodologia para um país como um todo, como no caso do Brasil, dada a sua grande extensão territorial (8,5 milhões de $\mathrm{km}^{2}$ ) e tamanho de sua população (aproximadamente 180 milhões de habitantes), é bastante complexo e difícil.

O objetivo deste trabalho é conhecer o padrão de mortalidade por causas maternas, a qualidade de sua informação e estimar a razão de mortalidade materna (RMM), bem como um fator de ajuste ou correção para os dados oficiais, no conjunto das capitais de Estados brasileiros e Distrito Federal, em cada uma das regiões brasileiras (Norte, Nordeste, Sudeste, Sul e Centro-Oeste) e no país como um todo.

\section{Metodologia}

O conjunto das 26 capitais de Estados e Distrito Federal apresentou, em 2000, uma população censitária igual a 41 milhões de habitantes, representando cerca de $24 \%$ da população brasileira; nessas mesmas áreas, a população feminina de 10 a 49 anos foi igual a 14.043 .856 habitantes, correspondendo a $25,3 \%$ do total de mulheres dessa faixa etária no Brasil $^{9}$.

Em 2000, o número de óbitos de mulheres de 10 a 49 anos no país foi de 63.821 casos, dos quais $17.414(27,3 \%)$ eram de residentes nas capitais brasileiras ${ }^{10}$.

Pelo fato de se acreditar não haver sazonalidade nas mortes maternas, para a presente investigação, foi tomada uma amostra de óbitos ocorridos no primeiro semestre de 2002.

Pela existência de heterogeneidade entre os contingentes populacionais das capitais e, por conseguinte, entre os seus totais de óbito, foi definido um tamanho mínimo para a amostra de mortes a serem analisadas, em função dos recursos e tempo disponíveis fixados pelo Ministério da Saúde. Após estimativas do possível número de mortes em 2002, ficou definido como período de referência do estudo aquele relativo aos meses de março, abril e maio de 2002, e ainda, como número mínimo a ser investigado, o total de cinqüenta mortes de mulheres de 10 a 49 anos em cada capital. Entretanto, para satisfazer este valor, foi preciso selecionar um número diferente de meses de ocorrência dos óbitos. Assim, para a grande maioria das capitais, as mortes foram referentes a três meses (março, abril e maio de 2002). Em algumas cidades foi preciso investigar todas as mortes ocorridas no primeiro semestre de 2002; em duas outras áreas, os óbitos referiam-se a quatro meses (fevereiro, março, abril e maio); no Rio de Janeiro e em São Paulo, dado o grande numero de residentes, a amostra de mortes foi relativa a dois meses. Por razões de ordem técnica, foram excluídas duas capitais do estudo (uma na Região Norte e outra na Região Nordeste).

A unidade amostral considerada foi o mês calendário, em função da pesquisa quantitativa, que pretendia comparar a totalidade dos óbitos de um mesmo mês, no hospital e nas outras fontes. Dessa maneira, foi investigado um total de 3.265 óbitos. 
Na apresentação e análise dos resultados finais para as cinco regiões e no âmbito do país como um todo, foi necessária uma padronização do período de referência, estimando-se então o número de óbitos de cada capital para os primeiros seis meses do ano. Assim, nas capitais em que os casos selecionados referiam-se a três meses, seus resultados foram duplicados; naquelas cidades em que foram coletados dados de quatro meses, o fator de multiplicação foi igual a 1,5; naquelas áreas onde os casos representavam as mortes de dois meses, o fator de ponderação foi igual a três; e naquelas capitais em que houve necessidade de coletar os óbitos ocorridos nos seis primeiros meses de 2002 não foi preciso usar um fator, pois já se referiam ao primeiro semestre. Com isso, a população de análise passou a ser igual a 7.332 mortes de mulheres de 10 a 49 anos, residentes nas capitais brasileiras e Distrito Federal, ocorridas no primeiro semestre de 2002.

Para cada caso foi feita uma entrevista no domicílio onde residia a mulher que havia falecido e preenchido um questionário, no qual constava a identificação completa da mulher, dados demográficos, composição da família, história das gestações, se estava grávida no momento da morte ou se esteve grávida nos 12 meses que a antecederam, atendimentos de pré-natal, atendimento médico, hospitalar, de Centro de Saúde e história da doença que levou a morte (autópsia verbal).

Após a entrevista familiar, foram feitas consultas com os médicos que cuidaram do caso, leitura dos respectivos prontuários médicos hospitalares, dos resultados de exames, dos laudos de autópsia, boletim de ocorrência policial (BO) e outras informações pertinentes.

De posse de todos esses dados, os formulários foram entregues a três técnicos (que haviam sido previamente calibrados) que estudavam a história clínica e, a partir dela, elaboravam uma nova declaração de óbito (DON). Depois, para cada caso, foram então codificadas as causas básicas e associadas das DO-N e, em outro momento, as causas das respectivas declarações originais (DO-O).
Com o resgate da informação, o conjunto das DO-N permitiu uma análise da mortalidade, a mais próxima possível da real, segundo causa básica e complicações da causa básica, incluindo as causas terminais e associadas.

No caso específico das mortes por causas maternas, a comparação entre as causas básicas especificadas nas DO-N e aquelas existentes nas DO-O possibilitou estimar um fator de correção ou de ajuste para o cálculo das RMM.

Em todas as capitais procurou-se conhecer também se havia morte de mulher de 10 a 49 anos sem o devido registro; foram encontrados apenas dois casos, ambos em capitais do Nordeste. Este dado foi obtido em função da metodologia que previa a comparação entre três fontes de dados, a saber: L1 (lista mensal de óbitos de residentes registrados nos cartórios), L-2 (lista mensal dos óbitos de mulheres residentes que morreram nos hospitais da capital) e L-3 (lista de óbitos de residentes e que foram autopsiados no Instituto Médico Legal) e elaboração de uma Lista Única, sem duplicações ou omissões, para ser comparada à Listagem de óbitos de residentes do Sistema de Informações sobre Mortalidade. Dessa forma, acredita-se que, se houve perda, refere-se apenas às mortes que ocorreram em domicílio e não foram registradas.

Para o cálculo das RMM, foram utilizados os valores referentes aos nascidos vivos no período estudado (primeiro semestre de 2002), provenientes do Sistema de Informações sobre Nascidos Vivos do Ministério da Saúde - SINASC ${ }^{11}$. Este sistema, tal qual o de registro de óbitos, também tem muito boa cobertura nas capitais de Estado $^{6}$.

A investigação foi coordenada, em nível central, por professores da Faculdade de Saúde Pública da Universidade de São Paulo; nas capitais de Estados, contou com um coordenador local e vários entrevistadores. No conjunto, trabalharam na pesquisa cerca de 300 pessoas.

O Projeto foi aprovado na íntegra pelo Comitê de Ética em Pesquisa da FSP/USP, e todos os familiares entrevistados assinaram termo de consentimento. 


\section{Resultados}

Os resultados mostram aspectos bastante interessantes e relevantes. Quanto às causas de morte de mulheres em idade fértil, as mais importantes foram, entre outras, os acidentes vasculares cerebrais; aids; causas externas, particularmente homicídios e suicídios; câncer de mama e de colo de útero - causas que estão sendo objeto de outras publicações.

Dos 7.332 óbitos, pôde ser constatado que $458(6,2 \%)$ ocorreram no aqui chamado ciclo gravídico puerperal ampliado (mortes ocorridas na gestação, no parto e até um ano após o mesmo). Essa proporção foi maior nas Regiões Norte $(8,4 \%)$ e Nordeste $(8,2 \%)$, em re- lação ao Sudeste (5,1\%), Sul (6,4\%) e CentroOeste $(6,4 \%)$. É digna de nota a ocorrência de cinco mortes verificadas mais de um ano após o parto, mas cuja causa básica foi uma causa materna; esses casos, segundo definições apresentadas na CID-10 $10^{1}$, são considerados “seqüelas de causa materna” (CID-10, Código O97), totalizando, portanto, 463 mortes. Destas, em 144 declarações de óbito originais já estava declarada, como causa básica da morte, uma causa materna, e em 319 estavam informadas outras causas. Com relação a estas últimas, a metodologia da Investigação permitiu detectar que 95 tornaram-se causas maternas e 224 continuaram como não maternas (Figura 1).

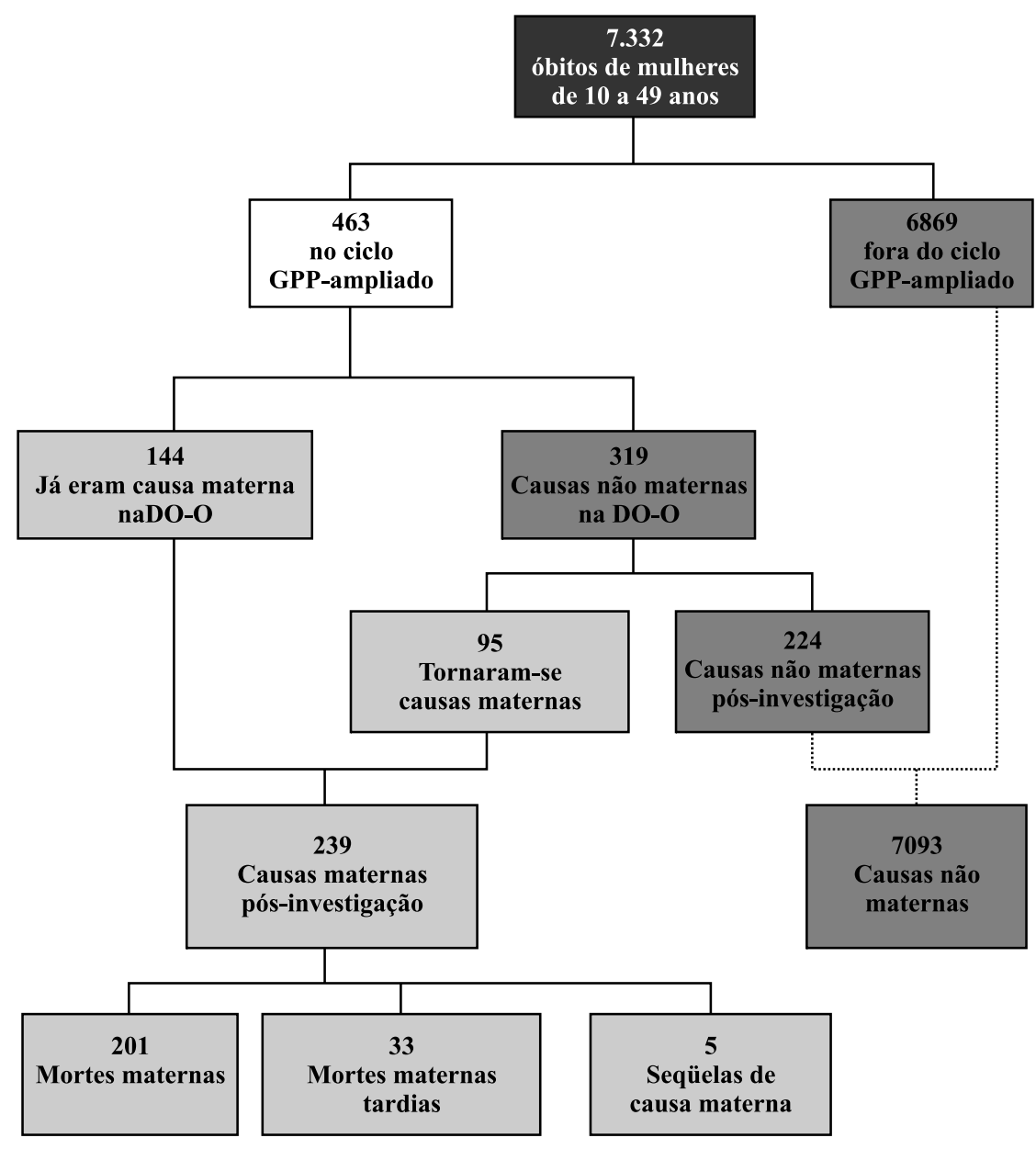

Figura 1 - Óbitos de mulheres de 10 a 49 anos segundo causa (materna ou não), conjunto de capitais brasileiras, $1^{\circ}$ semestre de 2002.

Figure 1 - Deaths of women between 10 and 49 years of age according to the underlying cause of death (maternal or not). Brazilian State Capitals, $1^{\text {st }}$ semester of 2002. 
Desta forma, após a pesquisa, foi possível identificar 239 casos em que a causa básica da morte era materna (Capítulo XV, CID-10), dos quais 201 óbitos ocorreram até 42 dias após o parto (mortes maternas) e 38, após esse período, sendo 33 causas maternas tardias (Código O96, CID-10) e 5 seqüelas de causas maternas (Código O97, na CID-10).

Com referência à distribuição dos 239 óbitos por causas maternas, segundo a situação obstétrica da mulher no momento da morte (gravidez, parto, puerpério e puerpério tardio), pôde ser observado que $22,6 \%$ ocorreram durante a gravidez e $28,9 \%$, nas primeiras 48 horas após o parto. O total considerado de mortes maternas, para o cálculo das RMM, foi de 201, representando $84,1 \%$ dos óbitos por causas maternas. Observando todas essas mortes ocorridas até 42 dias após o término da gestação, a proporção de gestantes foi de $26,9 \%$, o valor relativo às que estavam no puerpério de até 48 horas foi igual a $34,3 \%$, e $38,8 \%$ estavam no puerpério entre 48 horas e 42 dias (Tabela 1).

Comparando o número de mortes maternas (até 42 dias após o parto) declaradas na DO-O, o número de casos descobertos pela investigação e o total de casos, foi possível calcular fatores de correção ou de ajuste dessas mortes, para o Brasil e para as cin- co diferentes regiões do país, respectivamente, iguais a 1,4 e variando nas regiões entre 1,08 , para a Norte e 1,83 para a Região Sul (Tabela 2).

Foram estimadas também, para cada região e para o conjunto das capitais brasileiras, as respectivas taxas ou razões de mortalidade materna. As maiores foram observadas nas capitais das Regiões Norte e Nordeste e a menor na Região Sul, seguindo-se as relativas ao Sudeste e Centro-Oeste (Tabela 3).

Observando os óbitos maternos, isto é, aqueles ocorridos até 42 dias após o parto, segundo o tipo de causa, verificou-se que $67,1 \%$ constituíram-se em causas obstétricas diretas (Figura 2).

Quanto a algumas características das mulheres no ciclo gravídico puerperal (até 42 dias após o término da gestação) e cuja morte foi decorrente de causa materna, verificaram-se alguns aspectos interessantes.

Em relação às idades, embora se tenha propositadamente ampliado o período de fertilidade da mulher, aqui iniciado aos 10 anos, em virtude do elevado número de gestações na adolescência no país (em 2001, em $23,3 \%$ dos nascidos vivos, as mães eram menores de $20 \operatorname{anos}^{11}$ ), foi detectada apenas uma morte na idade de 14 anos. As idades

Tabela 1 - Número e proporção (\%) de óbitos por causas maternas: situação da mulher quanto ao binômio "gravidez x óbito", conjunto de capitais brasileiras, $1^{\circ}$ semestre de 2002. Table 1 - Number and proportion (\%) of deaths due to maternal causes according to status of women. Brazilian State Capitals, $1^{\text {st }}$ semester 2002.

\begin{tabular}{|c|c|c|c|}
\hline Situação no momento do óbito & $\mathrm{N}^{\circ}$ & $\%$ & $\% *$ \\
\hline Grávida & 54 & 22,6 & 26,9 \\
\hline \multicolumn{4}{|l|}{ No puerpério } \\
\hline$<48$ horas & 69 & 28,9 & 34,3 \\
\hline $48 \mathrm{~h}$ a 42 dias & 78 & 32,6 & 38,8 \\
\hline Subtotal & 147 & 61,5 & 73,1 \\
\hline Mortes Maternas (OMS) & 201 & 84,1 & 100,0 \\
\hline \multicolumn{4}{|l|}{ Parto há } \\
\hline 43 dias a 1 ano & 33 & 13,8 & \\
\hline 1 ano e mais & 5 & 2,1 & \\
\hline Total & 239 & 100,0 & \\
\hline
\end{tabular}

*A porcentagem foi calculada em relação ao total de óbitos ocorridos até 42 dias após o término da gestação.

* The percentage was calculated in relation to the total number of deaths that occurred up to 42 days after the end of pregnancy. 
Tabela 2 - Número de óbitos maternos na DO-Original e na DO-Nova e fator de ajuste segundo regiões, conjunto de capitais brasileiras, $1^{\circ}$ semestre de 2002.

Table 2 - Number of maternal deaths before (DO-O) and after (DO-N) investigation and adjusting factors according to Regions. Brazilian State Capitals, $7^{\text {st }}$ semester of 2002.

\begin{tabular}{lcccc}
\hline Regiões & DO-Original & DO-Nova* $^{*}$ & Total & $\begin{array}{c}\text { Fator } \\
\text { de Ajuste }\end{array}$ \\
\hline Norte & 25 & 2 & 27 & 1,08 \\
Nordeste & 38 & 29 & 67 & 1,76 \\
Sudeste & 55 & 19 & 74 & 1,35 \\
Sul & 6 & 5 & 11 & 1,83 \\
Centro-Oeste & 20 & 2 & 22 & 1,10 \\
\hline \multicolumn{1}{c}{ Brasil } & 144 & 57 & 201 & 1,40 \\
\hline
\end{tabular}

*descobertos pela investigação / *obtained by the investigation

Tabela 3 - Número de mortes maternas e razão de mortalidade materna* - RMM - segundo regiões, conjunto de capitais brasileiras, $1^{\circ}$ semestre de 2002.

Table 3 - Number of maternal deaths and maternal mortality ratio*-RMM-according to Regions.

Brazilian Capitals, $7^{\text {st }}$ semester of 2002.

\begin{tabular}{lcccc}
\hline Regiões & \multicolumn{2}{c}{ DO-Original } & \multicolumn{2}{c}{ DO-Nova } \\
& No & RMM & No & RMM \\
\hline Norte & 25 & 56,0 & 27 & 60,5 \\
Nordeste & 38 & 41,5 & 67 & 73,2 \\
Sudeste & 55 & 33,7 & 74 & 45,4 \\
Sul & 6 & 22,9 & 11 & 42,0 \\
Centro-Oeste & 20 & 44,8 & 22 & 49,3 \\
\hline \multicolumn{1}{c}{ Brasil } & 144 & 38,9 & 201 & 54,3 \\
\hline
\end{tabular}

* por 100.000 nascidos vivos / * per 100,000 live born

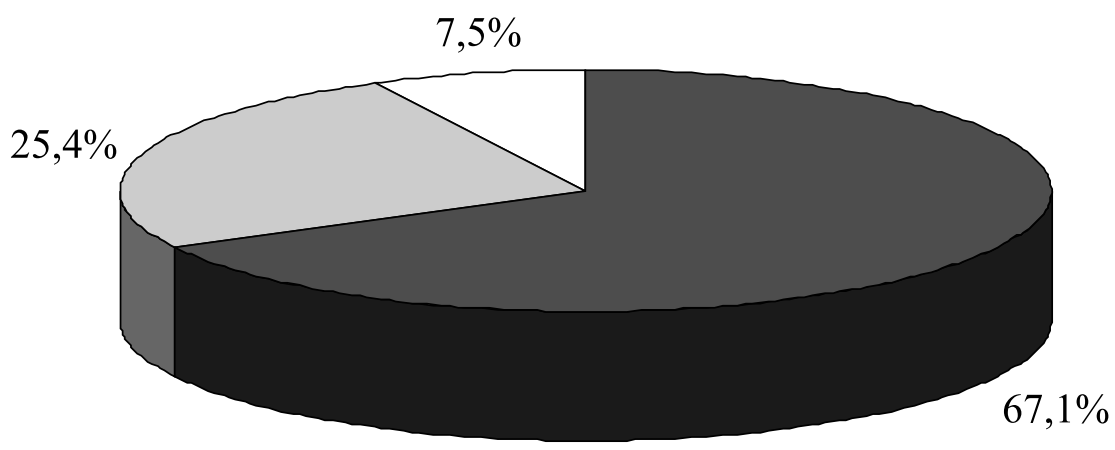

\section{$\square$ Diretas $\square$ Indiretas $\square$ Ignorado}

Figura 2 - Mortes maternas segundo tipo (diretas e indiretas), conjunto de capitais brasileiras, $1{ }^{\circ}$ semestre de 2002.

Figure 2 - Maternal deaths according to type of death (direct or indirect), Brazilian State Capitals, $1^{\text {st }}$ semester of 2002. 
média, mediana e máxima ao morrer dessas mulheres foram, respectivamente, 28,6 anos, 28 anos e 45 anos.

A distribuição das mulheres falecidas quanto à escolaridade mostra que $3,6 \%$ eram analfabetas, $53 \%$ cursaram até o primeiro grau incompleto, 25,6\% haviam concluído essa etapa; $8,9 \%$ completaram o segundo grau e $8,9 \%$ haviam freqüentado um curso superior. O estado civil revelou $25,4 \%$ de mulheres casadas e $32,8 \%$ vivendo em união consensual, sendo que as restantes $(41,8 \%)$ viviam sem companheiro (solteiras, viúvas ou separadas).

Quanto ao produto da última gestação, é digno de nota o fato de a informação não ter sido obtida em cerca de $40 \%$ dos casos. Daquelas mulheres em que foi possível conhecer o dado, $15,8 \%$ terminaram em aborto; em $19,2 \%$ o produto foi nascido morto e em $25,9 \%$ ela estava grávida no momento da morte, portanto sem expulsão do produto; no restante dos casos, o produto foi um nascido vivo.

\section{Discussão}

Com base na definição de morte materna, é possível o estabelecimento de dois outros conceitos, cuja distinção precisa ser feita: causas maternas e mortes maternas.

Assim, causas maternas "são todas aquelas causas ou diagnósticos incluídos no capítuloXVda CID-10 (os chamados códigos O)" . Mortes maternas, isto é, as consideradas para o cálculo da taxa/razão de mortalidade materna, referem-se às causas maternas, $\mathrm{ex}^{-}$ cluídas as causas codificadas em O96 e O97, acrescidas daquelas que não estão no capítulo XV da CID-10 e aparecem neste como "Exclusões", desde que a morte ocorra no período de até 42 dias após o parto, isto é, no chamado período puerperal .

A metodologia RAMOS ${ }^{8}$, empregada para investigar as mortes de mulheres de 10 a 49 anos, evidenciou que os óbitos por causas maternas estavam sub-declarados nas capitais brasileiras, comparando, de um lado, as causas descritas nas declarações de óbito originais (DO-O) e, de outro, o resgate da informação feito pela pesquisa (DO-N). Para a sua maior realidade, foi possível calcular um fator de correção ou de ajuste igual a 1,4, valor este bastante próximo daqueles descritos por Hill, AbouZahr e Wardlaw ${ }^{12}$ para 48 países com bom sistema de registro e relativamente boa declaração da causa de morte. Entretanto, pelos resultados aqui obtidos, ficou claro que a utilização de um único fator para o país como um todo não é recomendável, visto que o mesmo variou entre um mínimo de 1,08, na Região Norte, até um máximo de 1,83 na Região Sul.

A estimativa da Razão de Mortalidade Materna para o conjunto de capitais de Estados brasileiros e Distrito Federal, no primeiro semestre de 2002 , foi de 54,3 por 100.000 nascidos vivos. Esse valor é elevado, embora não pareça ser exageradamente alto. A experiência tem mostrado - e é essa a opinião de especialistas brasileiros, entre os quais se incluem os autores deste trabalho - que, no restante do país, a mortalidade não seria muito maior. Tanaka ${ }^{13}$, estudando a magnitude da mortalidade materna em 15 cidades brasileiras de três Estados (um no Norte, outro no Nordeste e um no Centro-Oeste do país), verificou não haver grandes diferenças entre os valores relativos às capitais e às cidades do interior. No Estado de São Paulo, já há alguns anos, a RMM para o interior do Estado é menor do que a da Capital e deve ser lembrado que, neste Estado, tanto a cobertura do registro de óbito é praticamente de $100 \%$, como a qualidade da declaração de óbito é boa. No Estado do Paraná, onde há um Comitê Central de Morte Materna e outros em cada um dos municípios, para avaliação e correção dos dados da mortalidade materna, observa-se que a RMM da capital, Curitiba, não difere muito da relativa ao restante do Estado ${ }^{5}$. Assim, para o total do país, é de se supor uma RMM não muito diferente dos valores encontrados para as capitais, valor esse menor do que os apontados por agências internacionais como WHO, UNICEF e UNFPA ${ }^{2}$. Para o Brasil, em 2001, a RMM calculada com os dados do SIM/MS e corrigida pelo fator 1,4 foi equivalente a 63,8 por 100.000 nascidos vivos $^{6}$. Esse fator $(1,4)$ foi adotado pelo Minis- 
tério da Saúde (Área Técnica de Saúde da Mulher) e pela RIPSA ${ }^{6}$, em 2004, como correspondendo ao fator de ajuste para o país, apesar de algumas críticas que ainda possam ser feitas ao mesmo.

Buerkens ${ }^{14}$, em editorial publicado no Bulletin of the World Health Organization, sob o título Is estimating maternal mortality useful?, faz comentários bastante interessantes a partir do trabalho de Hill, AbouZahr e Wardlaw $^{12}$ sobre estimativas da mortalidade materna, para 1995. Aponta várias limitações, concluindo, entretanto, que, apesar delas, as estimativas são úteis. Comenta que as disparidades entre as RMM de alguns países são tão gritantes que, mesmo com dados imprecisos, é possível observar sua persistência no tempo ou sua diminuição, fato que serviria para avaliar a atuação de programas e ações voltados à redução da mortalidade materna. Esses comentários são apropriados, mas há necessidade de se reafirmar não ser conveniente que, a partir de dados obtidos para uma localidade, estes venham a ser apresentados para o país como um todo, particularmente em áreas de grande extensão territorial e muita diversidade entre suas regiões, como é o caso do Brasil.

O estudo também deixou muito claro que é preciso investigar todas as mortes de mulheres em idade fértil, isto é, neste caso, de 10 a 49 anos, a fim de ser detectado o maior número possível de mortes maternas existentes. Algumas "Comissões ou Comitês de Estudo e Prevenção de Mortes Maternas” no país investigavam apenas as mortes maternas declaradas e as altamente suspeitas ou presumíveis. Na presente pesquisa, todavia, houve casos que se tornaram mortes maternas, apesar de terem seus atestados de óbito originais bem declarados com causa não materna, nem sequer suspeita. Nesse sentido, o Ministério da Saúde já determinou que esses Comitês passem a investigar todos os óbitos de mulheres de 10 a 49 anos, cujas causas possam ocultar um óbito materno ${ }^{15}$.

Com referência às doenças pré-existentes, aproximadamente a metade das famílias das falecidas informou sua presença. Sobressaíram-se, em freqüência, doenças do aparelho circulatório (60\%), sendo que a hipertensão, sozinha, esteve presente em 32,5\% dos agravos citados. Seguiram-se as doenças infecciosas, em 10,5\% das citações. É importante salientar que, segundo as famílias, $17,4 \%$ das falecidas eram hipertensas. Essas informações são extremamente relevantes e mostram o papel que um bom serviço de pré-natal pode desempenhar, prevenindo, tratando desses agravos e, quem sabe, até evitando essas mortes.

Quanto às causas de morte, por definição, o conjunto de mortes maternas (ou obstétricas) pode ser dividido em mortes obstétricas diretas e indiretas. As primeiras são aquelas devidas a complicações de causas ou estados que só ocorrem no ciclo gravídico puerperal - por exemplo, a eclâmpsia, a hemorragia por atonia uterina pós-parto, desprendimento prematuro de placenta, entre outros. As mortes maternas obstétricas indiretas são aquelas resultantes de doenças pré-existentes ou que se desenvolvem durante a gravidez, não devidas a causas obstétricas diretas, mas que foram agravadas pelo efeito fisiológico da gravidez ${ }^{1}$.

A presente pesquisa apontou que $67,1 \%$ das mortes maternas foram decorrentes de causas obstétricas diretas. Quanto aos diagnósticos específicos, predominaram os transtornos hipertensivos, com quase $25 \%$, praticamente a mesma proporção correspondente às causas obstétricas indiretas. Somente a doença hipertensiva específica da gravidez (pré-eclampsia e eclampsia) totalizou $37 \%$ de todas as mortes obstétricas diretas. As complicações hemorrágicas, particularmente devidas a placenta prévia e descolamento prematuro da placenta, e outras hemorragias foram responsáveis por $9,0 \%$ do total e 13,3\% das mortes obstétricas diretas (Tabela 4). Esses dados indicam que a melhoria da assistência é fundamental.

Embora no Brasil mais de 90\% dos partos ocorram em hospitais ${ }^{6}$ e, em algumas regiões, cheguem praticamente a $100 \%$, está claro que o que precisa melhorar - e muito é a assistência, aí incluídos os cuidados de pré-natal e uma adequada atenção ao parto e ao puerpério imediato. 
Tabela 4 - Número e proporção (\%) de mortes maternas segundo diagnósticos e regiões, conjunto de capitais brasileiras, $1^{\circ}$ semestre de 2002.

Table 4 - Number and proportion (\%) of maternal deaths according to diagnoses and Regions. Brazilian State Capitals, $1^{\text {st }}$ semester of 2002.

\begin{tabular}{|c|c|c|c|c|c|c|c|c|c|c|c|c|}
\hline \multirow[t]{2}{*}{ Diagnósticos } & \multicolumn{2}{|c|}{ Norte } & \multicolumn{2}{|c|}{ Nordeste } & \multicolumn{2}{|c|}{ Sudeste } & \multicolumn{2}{|c|}{ Sul } & \multicolumn{2}{|c|}{ C. Oeste } & \multicolumn{2}{|c|}{ Brasil } \\
\hline & No & $\%$ & No & $\%$ & No & $\%$ & $\mathrm{~N}^{\circ}$ & $\%$ & No & $\%$ & No & $\%$ \\
\hline Aborto (O00-O08) & 3 & 11,1 & 10 & 14,9 & 6 & 8,1 & 2 & 18,2 & 2 & 9,1 & 23 & 11,4 \\
\hline $\begin{array}{l}\text { Transtornos hipertensivos } \\
\text { (010-016) }\end{array}$ & 6 & 22,2 & 18 & 26,9 & 18 & 24,3 & - & - & 8 & 36,3 & 50 & 24,9 \\
\hline $\begin{array}{l}\text { Outros transtornos relacio- } \\
\text { nados à gravidez }(\mathrm{O} 20-\mathrm{O} 29)\end{array}$ & - & - & - & - & 6 & 8,1 & - & - & - & - & 6 & 3,0 \\
\hline $\begin{array}{l}\text { Problemas ligados ao feto, } \\
\text { membranas e placenta } \\
\text { (O30-048) }\end{array}$ & 2 & 7,4 & 8 & 11,9 & 6 & 8,1 & 2 & 18,2 & - & - & 18 & 9,0 \\
\hline $\begin{array}{l}\text { Complicações do trabalho } \\
\text { de parto e do parto } \\
\text { (O60-075) }\end{array}$ & 4 & 14,8 & 8 & 11,9 & 9 & 12,2 & - & - & - & - & 21 & 10,4 \\
\hline $\begin{array}{l}\text { Complicações do puerpério } \\
\text { (085-092) }\end{array}$ & 4 & 14,8 & 2 & 3,0 & 5 & 6,8 & 2 & 18,2 & 4 & 18,2 & 17 & 8,4 \\
\hline $\begin{array}{l}\text { Causa não especificada } \\
\text { (O95) }\end{array}$ & 1 & 3,7 & 6 & 9,0 & 6 & 8,1 & - & - & 2 & 9,1 & 15 & 7,5 \\
\hline $\begin{array}{l}\text { Causas obstétricas indiretas } \\
\text { (098-099) }\end{array}$ & 7 & 25,9 & 15 & 22,4 & 18 & 24,3 & 5 & 45,5 & 6 & 27,3 & 51 & 25,4 \\
\hline Total & 27 & 100,0 & 67 & 100,0 & 74 & 100,0 & 11 & $100,0^{*}$ & 22 & 100,0 & 201 & 100,0 \\
\hline
\end{tabular}

* aproximado para $100 \%$ / adjusted to $100 \%$

Dados do SINASC mostram que, em cerca de $47 \%$ dos nascidos vivos, no Brasil, a mãe tinha realizado sete ou mais consultas de pré-natal. Cabe então questionar a qualidade deste atendimento, já que, em quantidade, o que preceitua o Ministério da Saúde parece estar sendo atendido.

\section{Considerações finais}

No Brasil, além desta, que foi de âmbito nacional (apesar de se tratar somente de capitais), algumas investigações têm sido realizadas com o objetivo de corrigir os dados oficiais sobre mortes maternas ${ }^{7,16}$. A própria OMS tem recomendado a criação de novos métodos, com o objetivo de estimar, de for- ma mais fidedigna, a mortalidade materna.

No entanto, é preciso não perder de vista que o uso dessas estimativas deve ter caráter temporário. O que necessita ser, realmente, estimulado é o aprimoramento do sistema de informação em mortalidade, incluindo o melhor preenchimento da declaração médica da causa de morte ${ }^{17}$.

Nesse sentido, a gratuidade dos registros civis e, a partir de janeiro de 1998, a introdução de mais uma via na declaração de óbito, com a sugestão de busca ativa nas unidades notificadoras, a recomendação de utilização de dados de nascimentos e mortes informados pelo Programa de Saúde da Família ${ }^{18}$, entre outras, foram medidas válidas para se obter um aumento da captação dos eventos 
vitais. Por outro lado, visando a melhoria da qualidade da informação, a partir de alguns trabalhos ${ }^{7,19}$ e com base em proposta da OMS, feita na XXXIII ${ }^{a}$ Assembléia Mundial da Saúde, foi introduzida na declaração de óbito, no Brasil, a partir de 1996, variável relativa ao fato de a mulher estar grávida no momento da morte ou ter estado grávida nos doze meses que precederam o evento fatal (pergunta a ser feita em todos os casos de óbito de mulher em idade fértil). Foi feita uma primeira avaliação do preenchimento dessa variável, mostrando ausência de informação em cerca de $90 \%$ para ambas as perguntas $^{20}$, inclusive em casos em que a morte já era declarada como materna.

Nesta Investigação, procedeu-se à nova avaliação da variável, constatando-se que a mesma estava em branco, em cerca de $50 \%$ dos casos de óbitos de mulheres que se encontravam no ciclo gravídico puerperal. Tal achado pode talvez revelar uma possível melhoria da informação.

É preciso ficar claro, entretanto, que o simples preenchimento da variável, anotando a presença da gravidez, não remete automaticamente à ocorrência de morte materna. A informação deve ser vista como um indicativo de possível morte materna, a ser elucidada, sempre, por meio de investigação.

Quanto ao cálculo da Razão de Mortalidade Materna, é importante lembrar que a OMS, para comparações internacionais, propõe sempre o seu cálculo levando em conta as mortes verificadas durante a gestação e dentro dos 42 dias de puerpério. Porém, para propósitos nacionais, pode ser calculada uma outra razão, em que se considerem, também, as mortes ocorridas no puerpério tardio (além de 42 dias após o término da gestação).

Nesse sentido, foi proposto à OMS, que o capítulo XV da CID-10, "Gravidez, parto e puerpério”, a exemplo do que ocorre no capítulo XVI (Algumas afecções originadas no período perinatal), passasse a englobar todas as afecções originadas na Gravidez, parto e puerpério, independentemente do momento em que a morte viesse a ocorrer.

A consecução dos Objetivos do Desenvolvimento do Milênio ${ }^{3}$, no que se refere à meta de redução em três quartos da razão de mortalidade materna (entre 1990 e 2015), é representada, como se viu, por um conjunto de ações. Estas constituem-se em verdadeiros desafios, entre os quais quantificar o problema é apenas um deles.

\section{Referências}

1. World Health Organization. Classificação Estatística Internacional de Doenças e Problemas Relacionados à Saúde; 10a Revisão. CBCD, São Paulo; 1995.

2. WHO, UNICEF and UNFPA. Maternal mortality in 2000: Estimates developed by WHO, UNICEF and UNFPA; 2002.

3. United Nations - Department of Public Information. We the peoples. The role of the United Nations in the $21^{\text {st }}$ Century. New York; 2000 (disponível em www.un.org/millenium).

4. Ministério da Saúde/Secretaria de Políticas de Saúde/ Área Técnica de Saúde da Mulher. Manual dos Comitês de Mortalidade Materna, 2a Edição. Brasília; 2002.

5. Comitê Estadual de Prevenção da Mortalidade Materna. Estudo de casos de óbitos maternos. Paraná; 2002.
6. RIPSA - Rede Interagencial de Informações para a Saúde. Indicadores e Dados Básicos. Brasil; 2002. (disponível em www.datasus.gov.br).

7. Laurenti R, Buchalla CM, Lolio CA, Santos AH, Mello Jorge MH.. Mortalidade de mulheres em idade fértil no Município de São Paulo (Brasil), 1986. Rev Saúde Pública 1990; 24: 128-33.

8. Bouvier-Colle MH, Varnoux N, Costes P, Hatton F. Reasons for the underreporting of maternal mortality in France, as indicated by a survey of all deaths of women of childbearing age. Int J Epidemiol 1991; 20: 717-21.

9. IBGE (Fundação Instituto Brasileiro de Geografia e Estatística) (disponível em www.ibge.gov.br).

10. SIM/MS - Sistema de Informações sobre Mortalidade. Ministério da Saúde, Brasil; 2004 (disponível em www.datasus.gov.br). 
11. SINASC - Sistema de Informações sobre Nascidos Vivos. Ministério da Saúde, Brasil; 2004 (disponível em www.datasus.gov.br).

12. Hill K, AbouZahr C, Wardlaw T. Estimates of maternal mortality for 1995. Bull World Health Organ 2001; 79(3): 182-93.

13. Tanaka AC d'A, Mitsuiki L. Estudo da magnitude da mortalidade materna em 15 cidades brasileiras. São Paulo; 1999 (Pesquisa financiada pelo UNICEF).

14. Buerkens P. Editorial. Bull World Health Organ 2001; 79(3): 179.

15. Resolução do Ministério da Saúde - Área Saúde da Mulher; 2003 (disponível em http:// portalweb01.saúde.gov.br/saúde/visão).

16. Cardoso Silveira JA, Cecatti JG, Palma MM, Batista Filho M. Mortalidade materna pelo "Método das Irmãs" no Estado de Sergipe, Brasil. Revista do IMIP 1996; 10 (2): 97-102.
17. Laurenti R, Mello Jorge MHP, Gotlieb SLD. Reflexões sobre a mensuração da mortalidade materna. Cad Saúde Pública 2000; 16: 1.

18. Mello Jorge MHP, Gotlieb SLD. O Sistema de Informação de Atenção Básica como fonte de dados para os Sistemas de Informações sobre Mortalidade e sobre Nascidos vivos. Informe Epidemiológico do SUS 2001; 10(1): 7-18.

19. Laurenti R. Maternal mortality in Latin American urban areas: The case of São Paulo, Brazil. Bull PAHO 1993; 27(3): 205-14.

20. Laurenti R, Mello Jorge MHP, Gotlieb SLD. Mortes maternas no Brasil: análise do preenchimento da variável da declaração de óbito. Informe Epidemiológico do SUS 2000; 9(1): 43-50.

recebido em: 27/09/2004

versão final apresentada em: 09/11/2004

aprovado em: 08/12/2004 